Revista de Comunicación y Salud, 2020, Vol. 10, no 2, pp. 525-547

Editado por Cátedra de Comunicación y Salud

ISSN: 2173-1675

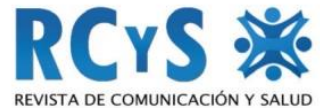

Enviado 15/08/2020

Aprobado 23/09/2020

\title{
GESTIÓN DE LA COMUNICACIÓN INTERNA Y EXTERNA EN INSTITUCIONES HOSPITALARIAS PRIVADAS EN ESPAÑA DURANTE LA CRISIS DEL COVID-19
}

\author{
Management of internal and external communication in private hospitals in Spain \\ during the COVID-19 crisis
}

Andrea Castro-Martínez

Universidad de Málaga. España.

andreacastro@uma.es

Pablo Díaz-Morilla

EADE Estudios Universitarios-University of Wales Trinity Saint David y Universidad de

Málaga. España.

pablodmorilla@uma.es

\section{Resumen}

Este trabajo analiza las estrategias comunicativas que han empleado las instituciones hospitalarias privadas en España durante la crisis del COVID-19, así como las herramientas que han aplicado para alcanzar a sus públicos internos y externos. Mediante un cuestionario compuesto por 52 preguntas se han recabado datos de 13 de los mejores hospitales según el ranking Merco 2020. Los resultados muestran que la composición de los departamentos de Comunicación es variada y que pertenecen a distintas áreas dentro de las instituciones. Las herramientas empleadas habitualmente tanto en comunicación interna como externa se han visto ampliadas para hacer frente a la pandemia y se han creado nuevos contenidos adaptados a los diferentes públicos con el objetivo de mantenerse como fuente fiable de información sobre la enfermedad y retener la confianza tanto de empleados como de pacientes. Las conclusiones indican que la crisis sanitaria ha transformado las rutinas de trabajo de los departamentos de comunicación interna y externa y que les ha servido para ganar agilidad, inmediatez y capacidad de reacción. La gestión estratégica de la comunicación de crisis ha sido fundamental en el ámbito hospitalario privado para afrontar con éxito la pandemia y lo será en el futuro para abordar los retos que traiga consigo la Nueva Normalidad.

Palabras clave: comunicación, crisis, relaciones públicas, salud, COVID-19, hospitales, comunicación interna, comunicación externa. 
Gestión de la comunicación interna y externa en instituciones hospitalarias privadas en España durante la crisis del COVID-19

\begin{abstract}
This paper analyses the communication strategies that private hospital institutions in Spain have employed during the COVID-19 crisis, as well as the tools they have applied to reach their internal and external audiences. Through a questionnaire composed of 52 questions, data has been collected from 13 of the best hospitals according to the Merco 2020 ranking. The results show that the composition of the Communication departments is varied and that they belong to different areas within the institutions. The tools commonly used in both internal and external communication have been expanded to deal with the pandemic and new content has been created, adapted to different audiences, with the aim of remaining a reliable source of information about the disease and retaining the trust of both employees and patients. The conclusions indicate that the health crisis has transformed the work routines of the internal and external communication departments and that it has helped them to become more agile, immediate and reactive. The strategic management of crisis communication has been fundamental in the private hospital environment to successfully confront the pandemic and will be fundamental in the future to address the challenges brought about by the New Normality.
\end{abstract}

Keywords: communication, crisis, public relations, health, COVID-19, hospitals.

\title{
Cómo citar el artículo
}

Castro-Martínez, A. y Díaz-Morilla, P. (2020). Gestión de la comunicación interna y externa en instituciones hospitalarias privadas en España durante la crisis del COVID-

19. Revista de Comunicación y Salud, 10 (2), 525-547. doi: https://doi.org/10.35669/rcys.2020.10(2).525-547

\section{INTRODUCCIÓN}

La pandemia del COVID-19 ha supuesto una crisis mundial con un alto impacto no solo en la economía sino en los sistemas de salud de todos los países (Andrikopoulos \& Johnson, 2020) y "ha provocado cambios dramáticos en el entorno de la atención clínica" (Shalowitz, Lefkowits, Landrum, Von Gruenigen \& Spillman, 2020 p.1). Esto ha obligado a los sistemas de atención sanitaria a reajustar sus estrategias y recursos, y a adaptar las áreas hospitalarias a la nueva situación (Santillán-Doherty, 2020), con medidas como la gran expansión de la telemedicina (Voulgaris, Ferini-Strambi \& Steiropoulos, 2020). La situación sin precedentes ha obligado a los profesionales de la salud a trabajar y comunicarse en un contexto de gran incertidumbre (Carico, Sheppard \& Thomas, 2020).

Para Mira (2020), el Sistema Público de Salud debe planificar el escenario poscrisis para lograr una vuelta ordenada a la normalidad, lo que implica prestar atención, entre otros temas, a las medidas para identificar cambios organizativos, así como reconocer y compensar el esfuerzo colectivo del personal sanitario, clave para afrontar la situación y que ha sufrido grandes daños físicos y emocionales. Esta planificación estratégica 
Gestión de la comunicación interna y externa en instituciones hospitalarias privadas en España durante la crisis del COVID-19

encaminada a superar los efectos de la pandemia en el sector público también se hace necesaria en las instituciones hospitalarias privadas. El cuidado de la salud mental y el desarrollo de habilidades de comunicación se han revelado como acciones necesarias para los profesionales sanitarios, que continuamente abordan situaciones nuevas en el contexto actual (Grover et al., 2020). Las organizaciones sanitarias se enfocan al liderazgo descentralizado y participativo para "asegurar una comunicación óptima dentro de la organización sanitaria y consolidar la motivación, formación y concienciación de los profesionales con respecto a su papel, objetivos y funciones" (Danet, García y March, 2016, p.92).

De este modo, es imprescindible mantener la excelencia en el cuidado clínico y en los procesos de comunicación y documentación, tal y como sucede normalmente en los departamentos de urgencias sanitarias, para reducir así tanto la posibilidad de mala praxis como otros riesgos (Maniya \& McGreevy, 2020). Una estrategia comunicativa adecuada permite gestionar una crisis sanitaria sin generar alarma social, como ocurrió en el caso de fiebre hemorrágica de Crimea-Congo en España en 2016 (Mestre y Bote, 2018).

\subsection{La comunicación durante la crisis del COVID-19.}

Los brotes de enfermedades infecciosas emergentes han aumentado notablemente en los últimos años - como el SARS o la gripe H5N1- y han puesto de manifiesto la relevancia de mantener comunicaciones eficaces para su gestión (De Sa, Mounier-Jack \& Coker, 2009). Para la Organización Mundial de la Salud (OMS) "la experiencia en materia de comunicación se ha vuelto tan esencial para el control de los brotes como la capacitación epidemiológica y los análisis de laboratorio" (World Health Organization, 2005, p.1). En este sentido hay dos conceptos relevantes: la comunicación del riesgo y la gestión del riesgo.

La comunicación del riesgo se refiere al proceso mediante el cual se identifica y comparte la información relativa a los brotes con el propósito de aumentar la conciencia de los principales interesados sobre la amenaza. Entre los principales interesados en las amenazas a la salud pública figuran la OMS, los gobiernos nacionales y el público. La gestión del riesgo, por el contrario, se refiere a los procesos de gestión asociados a la respuesta al brote, para los cuales la comunicación es crucial en cada etapa (De Sa, Mounier-Jack \& Coker, 2009, p.643).

La comunicación de crisis es un área de estudio específica que se centra en un "problema estratégico y de imagen que implica una crisis en una organización" (Micaletto, 2018) y se produce ante situaciones de riesgo elevado para la salud con la meta de que la población pueda sobrellevar los sentimientos negativos y superar los peligros que percibe (March, 2011). Una mala gestión puede conllevar consecuencias muy negativas, como sucedió en la crisis del Ebola en España (Micaletto y Gallardo, 2015). De hecho, la gestión de la comunicación durante la crisis del Ébola en España ha centrado diversos estudios como el de González Pacanowski, Medina Aguerrebere e Iglesias García (2017), que plantea un modelo para optimizar el proceso de 
Gestión de la comunicación interna y externa en instituciones hospitalarias privadas en España durante la crisis del COVID-19

comunicación de urgencia en base a aspectos como: elaborar un plan de comunicación para enfermedades infecciosas graves; disponer de canales, planes de formación y argumentarios específicos para los públicos internos así como portavoces autorizados; generar contenidos informativos y formativos para los públicos generales; emplear canales y contenidos móviles; y poseer un protocolo de información de contagio.

Según Castillo (2010, p. 205) las crisis tienen características propias, pero poseen una serie de elementos unificadores: la imagen y reputación de la organización, así como sus públicos internos, se ven afectados; atraen la atención mediática y el interés de los poderes públicos; conllevan buena imagen de las víctimas; y generan sentimientos de sorpresa y urgencia. Existen pautas para gestionar la comunicación en estos casos, como tener la iniciativa (Almada, 2009), comunicar con credibilidad y confianza, asumir las responsabilidades (Barquero y Barquero, 2005), unificar las intervenciones en la figura de un portavoz, evitar las mentiras, el silencio (Luecke, 2005; Xifra, 2009) y la improvisación (Rodríguez, Marauri, Armentia y Marín, 2020).

La crisis sin precedentes del COVID-19 ha tenido consecuencias entre otros muchos aspectos en el ámbito comunicativo, ya que hay mucha información en rápida evolución. Incluso la OMS ha empleado el término Infodemia (Allahverdipour, 2020; Alfonso y Fernández, 2020) para referirse a un contexto en el que se ha producido una amplia difusión de información falsa y en el que el miedo y la inseguridad han tenido gran protagonismo. "A la compleja situación sanitaria, económica y social se han sumado desafíos derivados de la gestión de la información sobre la enfermedad, que ha aumentado exponencialmente, y que pueden afrontarse proporcionando información objetiva y bien fundamentada" (Aleixandre-Benavent, Castelló-Cogollos y ValderramaZurián, 2020). Las noticias falsas que circulan por redes sociales (Pérez-Dasilva, MesoAyerdi y Mendiguren-Galdospín, 2020) y los bulos (Salaverría et al., 2020) acrecientan la necesidad de contar con fuentes de información fiable.

En situaciones de crisis los públicos buscan activamente información a través de distintas vías según sus necesidades: si pretenden obtener información privilegiada recurren a medios sociales, pero si sus necesidades son educativas acuden a medios tradicionales (Lu \& Jin, 2020). El objetivo de la comunicación para la salud es modificar actitudes y comportamientos para mejorar la calidad de vida y la salud pública, para lo que puede utilizar mensajes informativos o basados en la educación-entretenimiento (Igartúa, 2011), es decir que empleen llamamientos racionales o emocionales, ya que "la percepción de riesgo en gran medida es un sentimiento" (Stajnolovic, 2015, p. 106).

En el caso del coronavirus la ciudadanía ha incrementado su consumo informativo y la frecuencia con la que se informa y mantiene un posicionamiento crítico hacia el contenido de los medios, influenciado por la línea editorial y con un innecesario tratamiento sensacionalista y alarmista (Masip et al., 2020). Los medios digitales han sido el soporte más empleado para publicar sobre la COVID y han resultado muy relevantes para cubrir las necesidades de información de la población (LázaroRodríguez y Herrera-Viedma, 2020). Según Igartua, Ortega-Mohedano y ArcilaCalderón (2020, p.9) "el consumo de información sanitaria especializada en internet, 
Gestión de la comunicación interna y externa en instituciones hospitalarias privadas en España durante la crisis del COVID-19

webs oficiales y a través del personal sanitario mejora el conocimiento percibido sobre el coronavirus".

\subsection{La comunicación durante la crisis en el ámbito hospitalario.}

En los contextos de crisis "la necesidad de estar presente en todo momento es un imperativo innegociable" (Losada, 2018, p.23). Este es el motivo por el cual las instituciones sanitarias deben disponer de un plan de crisis que sirva de guía para el comportamiento corporativo, así como deben también mantener relaciones estables con los medios de comunicación y una presencia continuada en las redes sociales, con el objetivo de minimizar el impacto de estas situaciones y facilitar las actuaciones y comunicaciones destinadas a controlarlas (Calvo-Calvo, 2016).

La comunicación en instituciones hospitalarias debe contemplar tanto el ámbito interno como el externo para poder gestionar de forma integral todas las necesidades de la organización y alcanzar a los públicos a los que se orienta: "A nivel institucional, los centros asistenciales y los hospitales públicos y privados necesitan comunicarse con sus clientes potenciales y/o efectivos, además de con sus públicos internos" (Sánchez, 2011, p.11). Resulta relevante implicar a los profesionales en la comunicación de la organización y aplicar de forma complementaria herramientas analógicas y digitales (Barquero Cabero, Rodríguez Terceño y Gonzálvez Vallés, 2018; Costa-Sánchez y López-García, 2020).

Así, poseer un sistema sólido de comunicación que facilite el intercambio de conocimientos contribuye a prevenir y amortiguar los efectos de los nuevos incidentes de salud pública, ya que los sanitarios implicados emplean su experiencia y sus redes informales, principalmente locales, apoyándose así en relaciones de confianza y credibilidad que les ayudan a adoptar decisiones y comunicarse durante los periodos de crisis (Sanford, Schwartz \& Khan, 2020).

La comunicación constituye "la piedra angular para que se produzcan los fenómenos de cambio necesarios para la gestión del conocimiento y la innovación en la organización hospitalaria obteniendo mejores resultados en productividad y satisfacción de los clientes externos e internos" (Prieto y Olmo, 2010, p.6). Además de la presencia constante en medios, el empleo de canales de comunicación social y el hecho de incluir a todos los interesados en las comunicaciones son medidas que contribuyen a asegurar una comunicación adecuada y eficaz de los riesgos, especialmente importante durante la gestión de pandemias como la del coronavirus, por su alta tasa de infección y morbilidad y la falta de tratamientos específicos (Abrams \& Greenhawt, 2020).

Instrumentos como las plataformas de videoconferencias y los grupos de mensajes telefónicos se han usado durante el periodo del COVID-19 para mantener la comunicación, la cohesión de la comunidad de sanitarios y la educación continua (Edwards et al., 2020). Respecto al uso de las redes sociales por parte de los centros hospitalarios en un contexto de crisis, las respuestas rápidas provocan mayor confianza en la institución (Huanga \& DiStaso, 2020). La actualización permanente de los medios digitales se hace necesaria para mantener el control de la situación (Martín, 2013, p.46)

Revista de Comunicación y Salud, 2020, Vol. 10, nº 2, pp. 525-547 
Gestión de la comunicación interna y externa en instituciones hospitalarias privadas en España durante la crisis del COVID-19

así como la expresión de simpatía hacia los públicos y la muestra de aprendizaje organizativo, que generan sentimientos positivos en los usuarios hacia las entidades durante las crisis (Zhao, Zhan, \& Ma, 2020). Así, las redes sociales permiten una comunicación de crisis proactiva que potencia la proximidad con los públicos, aunque en muchos organismos este canal no esté totalmente incorporado a la gestión de crisis (Calleja-Reina, Paniagua y Victoria, 2018).

En los últimos años se ha potenciado el desarrollo de la Comunicación Interna $(\mathrm{Cl})$ en las instituciones sanitarias (Costa, 2011), ya que "los públicos internos (empleados) se deberían constituir en uno de los públicos principales para las organizaciones de salud" (Bustamante, 2012, p.88). Para Medina (2012) la Cl en el ámbito hospitalario tiene un carácter estratégico al permitir alcanzar metas globales, ya que afecta a la satisfacción del paciente y, por tanto, a la imagen y reputación de la entidad, lo que justifica la creación de un departamento de Cl dentro de la organización. "La Comunicación en los centros asistenciales resulta fundamental para la convivencia y el trabajo eficiente, teniendo en cuenta la diversidad de perfiles profesionales con los que se cuentan y las múltiples líneas de mando y múltiples metas que coexisten en función de los grupos profesionales" (Sánchez, 2011, p.11). Para alcanzar sus objetivos, la $\mathrm{Cl}$ implica a todos los empleados y utiliza diversas estrategias y soportes de comunicación, como revistas corporativas o intranet (Medina, 2012). La creación de nuevos contenidos se vuelve indispensable para mantener la atención de los públicos, tal y como se ha hecho por parte de la sanidad pública en México y en España ante la crisis sanitaria del COVID-19 (Estrella, 2020).

Sin embargo, en el caso de la actual crisis no solo resulta clave la gestión de comunicación que realice cada institución a nivel individual, sino que la cooperación y comunicación interinstitucional continua es imprescindible para responder de modo eficaz ante una pandemia, ya que esto posibilita aplicar estrategias proactivas en lugar de reactivas (Kortepeter, Kwon, Christopher, Hewlett \& Cieslak, 2017). Es por ello por lo que en las crisis sanitarias "las administraciones deben actuar siempre bajo el criterio de alertar a la población de los riesgos a los que puede estar expuesta, pero, a la vez, intentando evitar alarmas innecesarias" (Rodríguez, 2011, p.42).

En las últimas décadas organismos públicos y empresas privadas se han interesado en comunicar la ciencia y realizar una labor divulgativa como parte de su política de relaciones públicas y de responsabilidad social corporativa (García-Hernández, Martínez-Rodrigo y Victoria, 2016). Este posicionamiento adquiere aún más consistencia durante la crisis del COVID-19, ya que los centros hospitalarios plantean su comunicación como maniobra de gestión de la situación y, al tiempo, como parte de su estrategia de relaciones públicas. Para Xifra $(2020$, p. 6) en el contexto de la actual pandemia "la gestión de la comunicación interna pasa a ser también una cuestión de responsabilidad social corporativa" y será clave para salvaguardar la seguridad y bienestar de las plantillas. Por todo ello, la comunicación durante una crisis determina la relación tanto con públicos internos como externos con el objetivo de generar confianza y credibilidad y contribuir a la resolución de la situación problemática (Acevedo, 2020).

Revista de Comunicación y Salud, 2020, Vol. 10, nº 2, pp. 525-547 
Gestión de la comunicación interna y externa en instituciones hospitalarias privadas en España durante la crisis del COVID-19

\section{OBJETIVOS}

El objetivo de esta investigación se centra en analizar el modo en que las instituciones hospitalarias del sector privado han afrontado la crisis del COVID-19 en el aspecto comunicativo. Para alcanzar este propósito se han planteado otros objetivos secundarios como son:

- Estudiar la composición de los departamentos de comunicación en estas organizaciones.

- Determinar qué herramientas de $\mathrm{Cl}$ y de CE utilizan normalmente estas instituciones y cuáles son las herramientas que han tenido mayor protagonismo durante la pandemia.

- Describir las estrategias comunicativas que han empleado durante este periodo las instituciones hospitalarias analizadas tanto hacia sus públicos internos como hacia la sociedad y públicos externos.

\section{METODOLOGÍA}

Esta investigación se enmarca en los estudios sobre comunicación y salud y, más específicamente, en la línea sobre comunicación estratégica en periodos de crisis sanitaria. En concreto se circunscribe al modo en que los centros hospitalarios del sector privado español han gestionado la comunicación, tanto a nivel interno como externo, durante la crisis provocada por el COVID-19.

Se ha optado por un diseño metodológico mixto (Teddlie \& Abbas, 2010) que permita obtener una visión más completa del fenómeno (Hernández, Fernández \& Baptista, 2010). La muestra la conforman 13 instituciones hospitalarias españolas pertenecientes al ranking de hospitales privados del Monitor de Reputación Sanitaria 2020 de Merco (Monitor Empresarial de Reputación Corporativa). Los datos ofrecidos por Merco constituyen una referencia en el sector y son habitualmente empleados para realizar investigaciones científicas (Costa-Sánchez, Túñez-López y Videla-Rodríguez, 2016; Mira, Lorenzo, Navarro, Guilabert y Pérez-Jover, 2015; Gost, 2015).

La herramienta para la obtención de la información ha sido un cuestionario (Wimmer \& Dominick, 1996) con 52 preguntas formuladas de manera concisa y clara con el fin de evitar errores de interpretación. El cuestionario se construye mediante preguntas cerradas y preguntas abiertas, que aportan un valor cualitativo a la investigación, y valoraciones a través de la escala de Likert. Las preguntas se dividen en tres bloques: el primero contiene aspectos sobre la organización y perfil sociodemográfico de su responsable de Comunicación (sexo, estudios, ámbito de especialización, remuneración...); el segundo aborda cuestiones sobre $\mathrm{Cl}$ en la institución hospitalaria en el escenario previo al COVID-19 y durante la pandemia (especialistas en comunicación interna, instrumentos y estrategias empleadas, plan de comunicación de crisis, herramientas aplicadas durante la crisis...); y el tercero se centra en la gestión de la CE en la organización antes y durante la pandemia (posición en el organigrama, 
composición del equipo de trabajo, estrategias y acciones empleadas, plan de crisis externo, herramientas utilizadas durante la pandemia...).

Tras elaborar la plantilla a través de Google Forms, se procedió al envío del cuestionario a los correos corporativos de los diferentes centros hospitalarios, con los que previamente se había contactado. También se les ofreció la posibilidad de responder en archivo de texto para solventar problemas de seguridad derivados del acceso a enlaces externos desde sus organizaciones.

La dirección de comunicación de los centros hospitalarios del grupo HLA rehusó la posibilidad de participar en la investigación, por lo que el Hospital Universitario HLA Moncloa, la Clínica Vistahermosa Grupo HLA, el Hospital HLA El Ángel y el Hospital HLA Jerez Puerta del Sur fueron descartados.

\section{RESULTADOS}

El tamaño de las plantillas de los centros que conforman la muestra es mayoritariamente grande, ya que el $69,2 \%$ tiene más de mil empleados, aunque también están presentes instituciones medianas y pequeñas, pues el 15,4\% tiene entre 251 y 500 trabajadores y el $15,4 \%$ tiene menos de 50 empleados. Respecto al volumen anual de pacientes que atienden todos superan la cifra de 20.000 personas.

El presupuesto que destinan a Comunicación es variable (gráfico 1) pero lo habitual es que supere los 100.000 euros. Los departamentos o equipos que gestionan la comunicación están formados de media por 4 personas y en su mayoría son hombres $(72,4 \%)$. Lo habitual es que tengan estudios de máster o posgrado $(66,7 \%)$ o de gradolicenciatura $(30,3 \%)$, mientras que el nivel de doctorado apenas tiene presencia (3\%). La especialidad de sus estudios es en la mayoría de los casos Comunicación, aunque aparece Marketing-Comunicación Comercial y en una menor medida Económicas-MBAGestión de organizaciones.

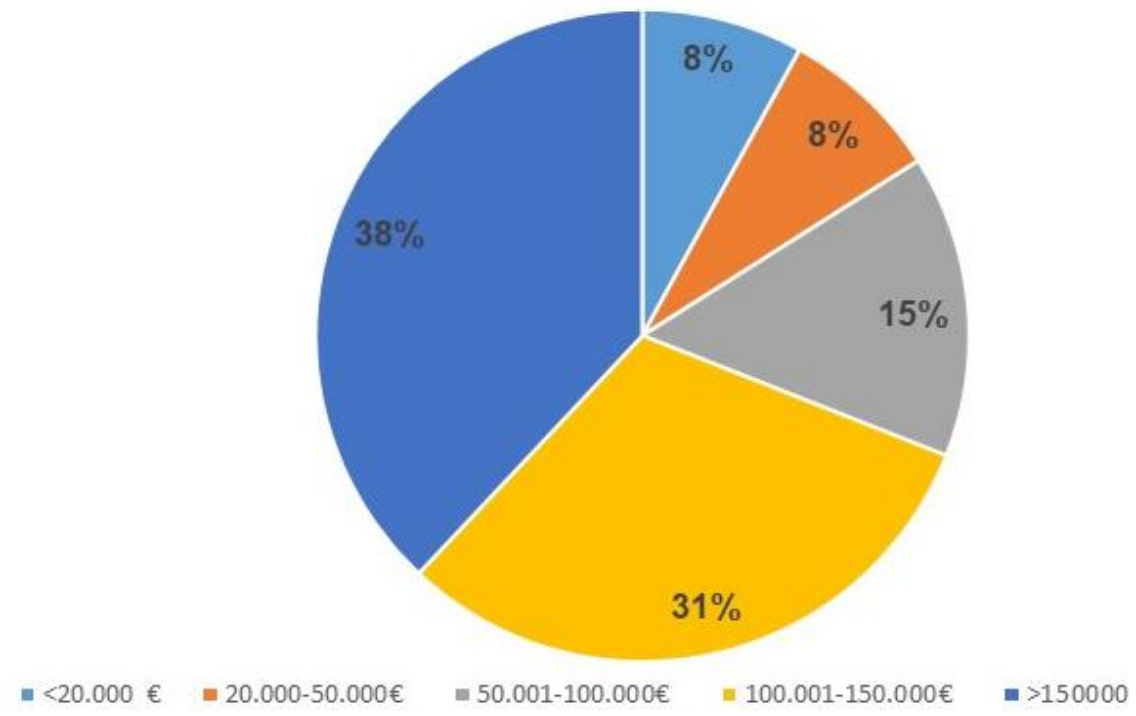

Revista de Comunicación y Salud, 2020, Vol. 10, no 2, pp. 525-547 
Gestión de la comunicación interna y externa en instituciones hospitalarias privadas en España durante la crisis del COVID-19

\section{Gráfico 1. Presupuesto anual destinado a Comunicación.}

Fuente: elaboración propia.

Respecto a la figura del Dircom en todos los casos el puesto está ocupado por hombres, aunque de edad variable, pues el $46,2 \%$ tienen entre 46 y 55 años; el 30,8\% entre 30 y 45 y el $23,1 \%$ entre 56 y 65 años. La posición que ocupa el director de Comunicación en la organización se vincula a diferentes áreas: Comunicación Corporativa (38,5\%), Dirección de Comunicación $(30,8 \%)$ y Corporate Affairs $(30,8 \%)$.

En cuanto a la formación de los Dircom, la especialidad principal de sus estudios es Comunicación (92,3\%), seguida de Marketing y Comunicación Comercial $(38,5 \%)$ y una presencia poco significativa de Periodismo $(7,7 \%)$; la mayoría $(69,2 \%)$ posee un máster o posgrado, el $23,1 \%$ es graduado o licenciado y solo el $7,7 \%$ alcanza el nivel de doctorado. El sueldo bruto anual que perciben oscila entre los rangos de $70.001 \mathrm{a}$ 100.000 euros $(38,5 \%)$ y de 20.000 a 40.000 euros $(15,4 \%)$, aunque la mayor parte $(46,2 \%)$ recibe una remuneración que se sitúa en la franja entre los 60.000 y 70.000 euros anuales.

Las actividades asociadas al puesto son muy variadas (gráfico 2) y entre ellas destacan la comunicación estratégica y la coordinación, las relaciones con los medios y los eventos. La comunicación de crisis tiene un papel importante, aunque no así la comunicación interna.

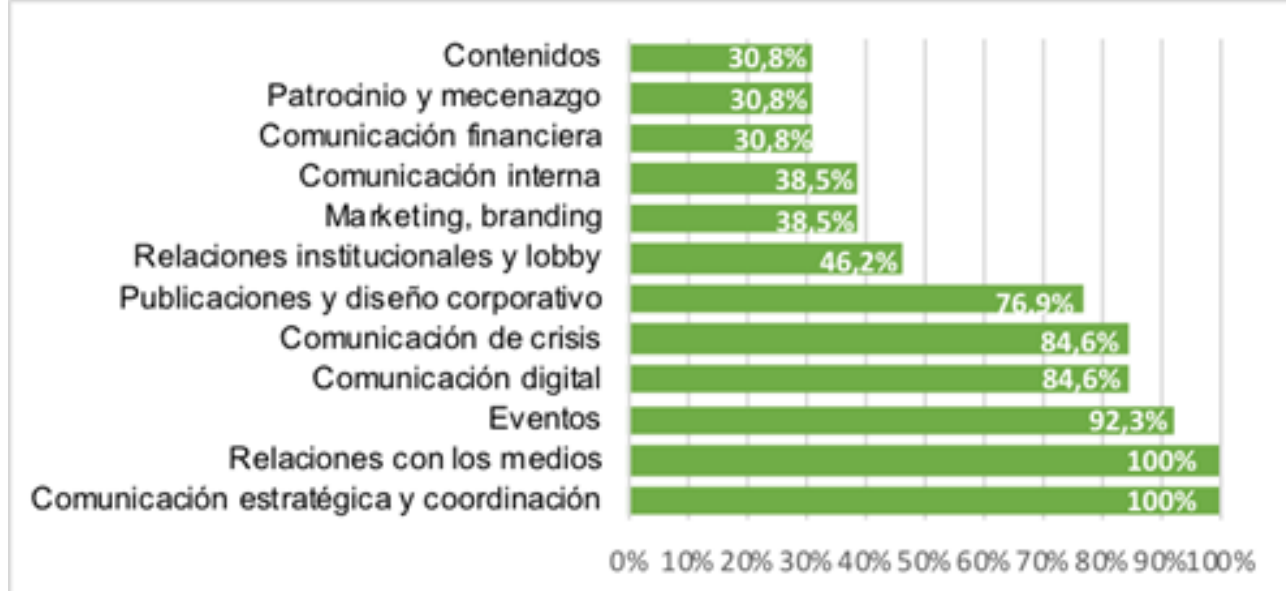

Gráfico 2. Actividades desarrolladas por el/la Dircom.

Fuente: elaboración propia.

La gestión de la $\mathrm{Cl}$ en la organización se sitúa en dependencia de diferentes áreas como la Dirección de Recursos Humanos (46,2\%), Corporate Affairs Executive Director $(30,8 \%)$ o la Dirección General (15,4\%). Es relevante destacar que tan solo en el 7,7\% de las organizaciones analizadas la $\mathrm{Cl}$ depende de la Dirección de Comunicación. El número medio de profesionales dedicados a este ámbito es de 2 , aunque en más de la mitad de los centros únicamente hay una persona que se encarga de la gestión de la Cl. 
La planificación de la $\mathrm{Cl}$ en las instituciones analizadas se encuentra muy polarizada entre las que disponen de un plan diseñado previamente al que incorporan acciones cuando lo estiman necesario $(53,8 \%)$ y aquellas que basan su $\mathrm{Cl}$ en acciones puntuales y esporádicas $(46,2 \%)$. Para desarrollar estas estrategias emplean herramientas muy variadas (gráfico 3). Combinan las tradicionales -como eventos, buzón de sugerencias, formación o manual de bienvenida- con instrumentos digitales y audiovisuales newsletter, intranet, blogs, videoconferencias, revistas digitales, vídeos, aplicaciones propias o realidad virtual- y en algunos casos con comunicación informal cara a cara con los empleados. Ninguna organización utiliza en su estrategia las revistas internas físicas, los podcasts, las redes sociales internas, la mensajería instantánea o el tablón de anuncios.

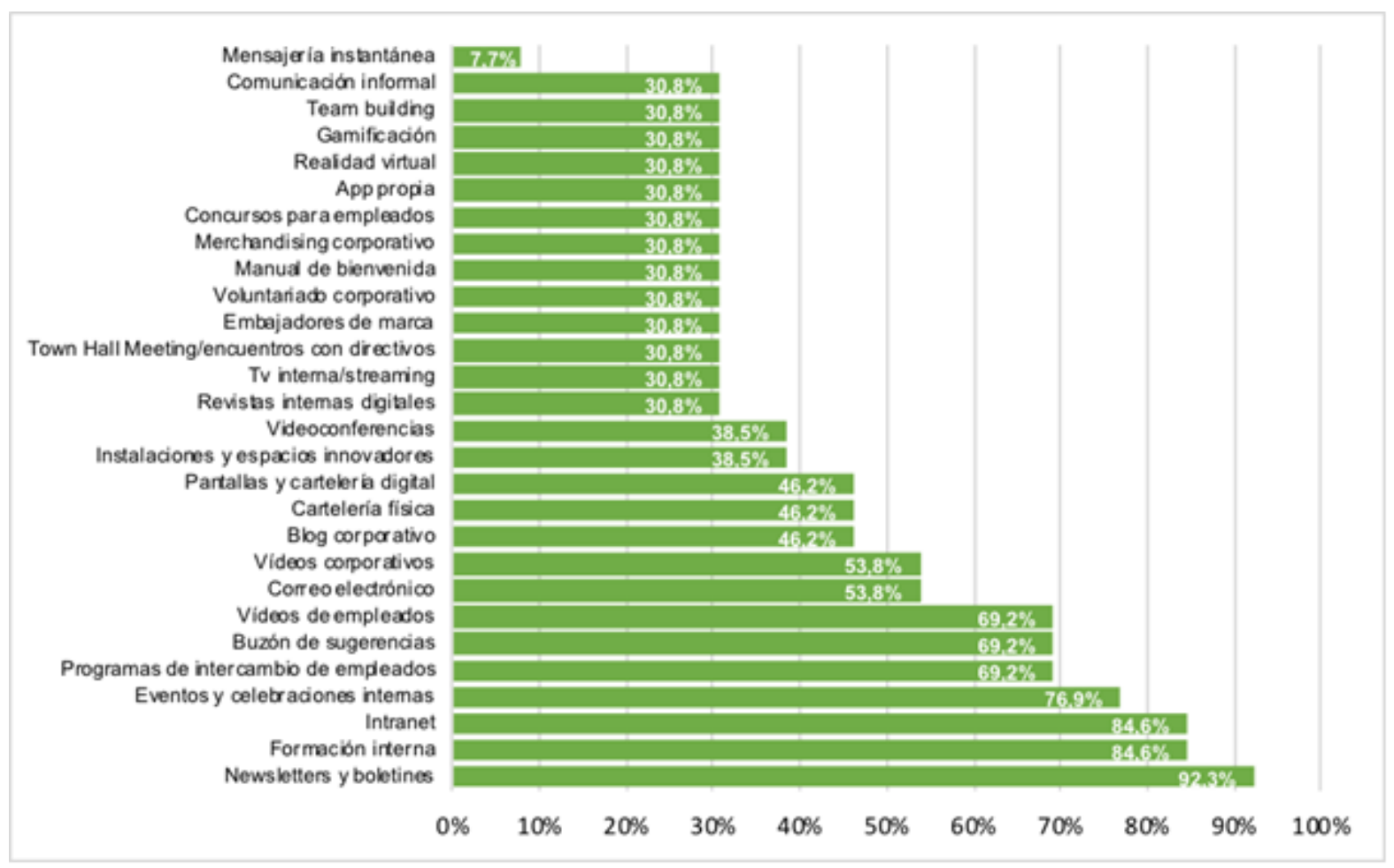

Gráfico 3. Herramientas de Cl empleadas en las organizaciones hospitalarias.

Fuente: elaboración propia.

La mayor parte de los centros $(76,9 \%)$ desarrolla campañas para potenciar el bienestar de sus plantillas en aspectos como la participación de los empleados, la escucha activa, la integración de la diversidad, fomento de la igualdad/apoyo al talento femenino, cuidado de la salud física y mental, retribución emocional y beneficios sociales, becas y ayudas económicas, posibilidad de teletrabajo, conciliación y racionalización de horarios, fomento de la práctica deportiva, disminución del estrés y desarrollo de espacios innovadores que fomenten la comunicación y el sentimiento de pertenencia. 
Gestión de la comunicación interna y externa en instituciones hospitalarias privadas en España durante la crisis del COVID-19

Como temas estratégicos de cara al futuro desarrollo de la $\mathrm{Cl}$ destacan entre otros: fortalecer los vínculos con la marca para potenciar la confianza en la organización (76,9\%); capacitación, formación interna y retención de talento $(76,9 \%)$; y crear y gestionar nuevos canales y contenidos internos (53,8\%). $Y$ es que todos los participantes consideran la $\mathrm{Cl}$ una pieza clave en la organización, en palabras de uno de ellos: "La comunicación interna es igual de estratégica que la externa. Sin una adecuada gestión del público interno, se puede romper la coherencia entre lo que eres y lo que cuentas que eres".

Las herramientas empleadas para la medición de resultados en $\mathrm{Cl}$ son principalmente cuatro: las reuniones con empleados (92,3\%), las encuestas internas de satisfacción y clima laboral $(76,9 \%)$, las auditorías externas de comunicación $(30,8 \%)$ y la monitorización de los distintos canales empleados $(30,8 \%)$.

La Cl se ha visto totalmente alterada por la crisis del COVID-19, una afirmación en la que coinciden todos los encuestados, pero ha supuesto un reto del que han aprendido valiosas lecciones. Algunos de sus comentarios al respecto son:

- "Nos ha puesto a prueba en el peor escenario que habíamos conocido. Contar con un plan específico de continuidad para este escenario nos ha ayudado a tener un punto de partida, pero ese plan ha evolucionado y crecido durante la pandemia para ajustar canales y mensajes".

- "Precisamente, ha reforzado la comunicación interna y la vinculación y adscripción del personal con la empresa".

- "Nos ha enseñado a sacar lo mejor de cada uno de los trabajadores y a generar sinergias entre los distintos departamentos".

Y es que, aunque la mayoría de los centros (77\%) disponía de un plan de crisis a nivel interno, únicamente el $30,8 \%$ de ellos contemplaba una situación tan extrema como la actual pandemia.

Todos coinciden en que la gestión de la Cl durante la crisis del COVID-19 ha sido totalmente distinta a la de otras crisis anteriores. Los principales aspectos diferenciadores han sido la intensidad, la inmediatez, la permanencia en el tiempo, la necesidad de respuestas diversas y constantes, el grado de incertidumbre y el distinto perfil de la actividad. Uno de los participantes lo explica del siguiente modo:

Todos nuestros médicos, enfermeras, técnicos, auxiliares, recepcionistas, personal de seguridad, de limpieza, etc., seguían viviendo la pandemia (y las incertidumbres asociadas) cuando regresaban a sus casas. Nuestra obligación era también acompañarles y ayudarles en esa esfera, a ellos y a sus familias, para poder entender y gestionar mejor la situación (niños pequeños, mayores a su cargo, la propia tensión emocional...).

La totalidad de los centros hospitalarios ha adoptado nuevas medidas en el ámbito de la $\mathrm{Cl}$ (gráfico 4) entre las que destacan la formación interna, el aumento de los equipos de protección, la escucha activa de las propuestas del personal y el apoyo psicológico a los empleados. 
Gestión de la comunicación interna y externa en instituciones hospitalarias privadas en España durante la crisis del COVID-19

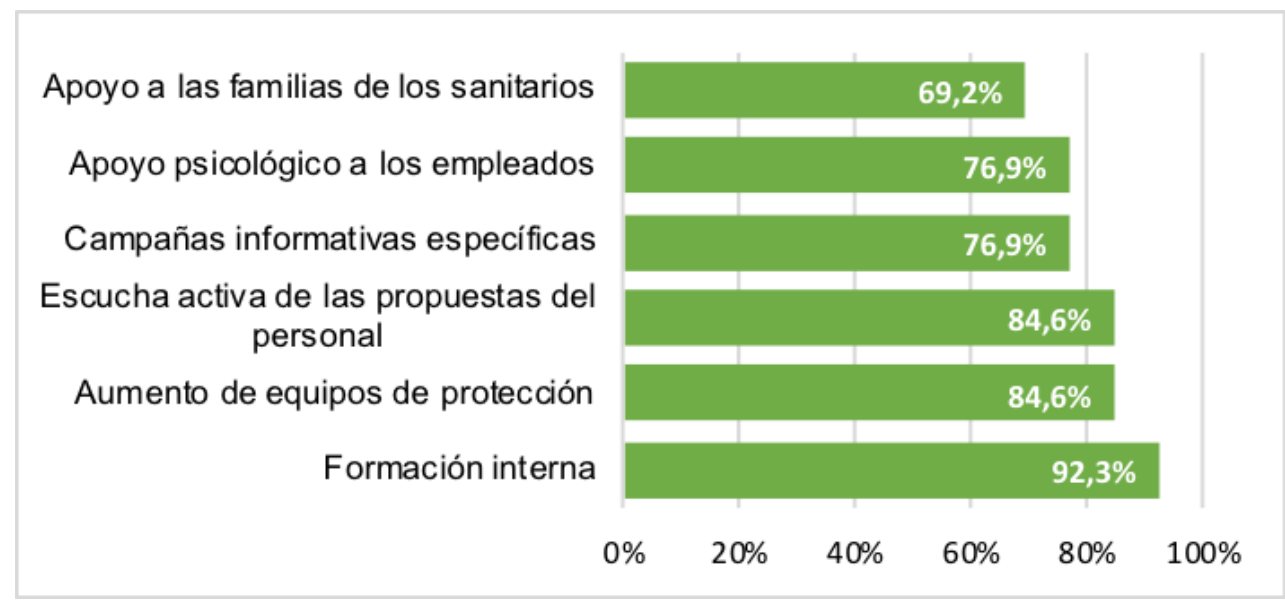

Gráfico 4. Medidas de Cl adoptadas durante la crisis del COVID-19.

Fuente: elaboración propia.

En todas las organizaciones se han creado durante la crisis materiales específicos de $\mathrm{Cl}$ adaptados a cada uno de los stakeholders (tabla 1) y las herramientas que más han empleado han sido la videoconferencia $(84,6 \%)$, el email $(61,5 \%)$, la mensajería instantánea -en concreto el servicio de WhatsApp- (53,8\%), la intranet y el teléfono $(46,2 \%)$, aplicaciones internas y canales de streaming $(30,8 \%)$ y las redes sociales internas $(7,7 \%)$.

Tabla 1. Materiales de Cl creados para gestionar la crisis del COVID-19 en los hospitales.

\begin{tabular}{|l|l|}
\hline Material & Tasa de uso \\
\hline Cartelería física y virtual & $93,2 \%$ \\
\hline Mensajes de la Dirección & $93,2 \%$ \\
\hline Notas y circulares & $76,9 \%$ \\
\hline Infografías & $69,2 \%$ \\
\hline Newsletter y emailing & $69,2 \%$ \\
\hline Vídeos formativos & $69,2 \%$ \\
\hline Vídeos informativos & $69,2 \%$ \\
\hline Vídeos motivacionales & $46,9 \%$ \\
\hline Folletos informativos & $38,5 \%$ \\
\hline
\end{tabular}

Fuente: elaboración propia.

Otro elemento crítico en la gestión de la $\mathrm{Cl}$ durante la crisis ha sido el papel de los directivos y jefes de equipo, que han tenido una participación muy activa en todos los casos, se han implicado en la coordinación de tareas y han tomado parte en las iniciativas propuestas. De esta forma se mantuvo la coherencia entre lo que la entidad manifiesta y lo que ellos transmiten a sus equipos. Los liderazgos locales han sido un factor de éxito en la gestión de la $\mathrm{Cl}$ durante la crisis, ya que consideran que es un tema crítico para la cultura organizacional en la que debe implicarse toda la compañía. 
El $100 \%$ de los participantes coincide en que la crisis ha modificado la forma de gestionar la $\mathrm{Cl}$ en las instituciones hospitalarias y que lo seguirá haciendo en el futuro. Ha influido en la relación y vinculación entre la organización y sus empleados y contribuido a que los equipos de $\mathrm{Cl}$ ganen inmediatez, agilidad y efectividad y sean conscientes de la importancia de que todos los mecanismos de comunicación funcionen correctamente. Además, les ha acercado a todas las áreas de gestión poniendo de manifiesto la necesidad de hacer más permeable el trabajo de los miembros de la entidad.

En cuanto a la Comunicación Externa (CE), se sitúa en dependencia de Comunicación Corporativa (38,5\%), Corporate Affaires (30,8\%), Dirección General $(23,1 \%)$ y Dirección de Comunicación $(7,7 \%)$. El número medio de integrantes de los departamentos es de 4,5 (el 46,5\% de las organizaciones tienen 4 personas dedicadas a esta área). En todos los casos la CE se gestiona mediante un plan diseñado y organizado previamente, aunque en el $92,3 \%$ de ellos se pueden incorporar acciones si se considera necesario. Entre las herramientas que más emplean (gráfico 5) destaca la relación con medios de comunicación, la web corporativa y el uso de las redes sociales. La medición de resultados no se produce en el 7,7\% de los casos, aunque lo habitual en el resto de entidades es que empleen diversos métodos como impacto en medios de comunicación y redes sociales, respuesta a eventos externos, medición de kpi especificados en el plan anual (penetración del mensaje en audiencias clave, reflejo en rankings de reputación, medición propia de reputación...), etc.

Las tendencias en el ámbito de la CE que identifican los encuestados se concretan en: potenciar la conversación para establecer relaciones más duraderas, de confianza, satisfacción y cercanía a través de una comunicación más amable y menos agresiva; difundir una actividad basada en la humanización; potenciar la participación de los usuarios para conocer sus opiniones y dar a conocer más los servicios del hospital, potenciando sus valores; y en el uso de plataformas digitales y soluciones on-line encaminadas aumentar la presencia de la marca.

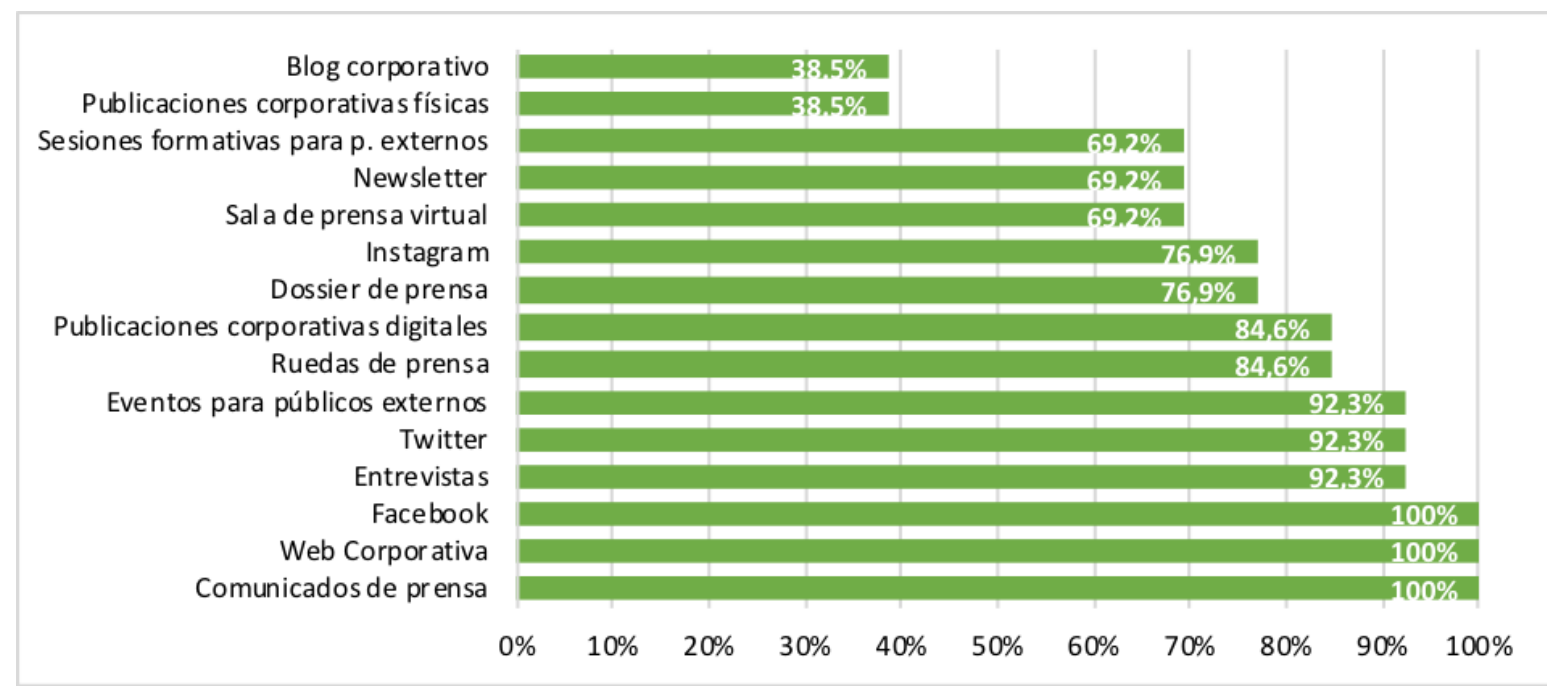

Gráfico 5. Herramientas de CE utilizadas en los centros hospitalarios.

Fuente: elaboración propia. 
Gestión de la comunicación interna y externa en instituciones hospitalarias privadas en España durante la crisis del COVID-19

En cuanto a la crisis del COVID-19, el 69,2\% de las organizaciones no disponían de un plan de crisis que contemplase un escenario de la magnitud actual. Los centros Sanitas ya habían incorporado en el Plan de Comunicación 2020 un anexo sobre pandemias ante las alertas previas de la OMS. Al igual que en el caso de la $\mathrm{Cl}$, la pandemia ha afectado a la CE ya que según afirma un participante "nos ha puesto a prueba en el escenario más hostil y de mayor incertidumbre que habíamos vivido". De hecho, todas las respuestas señalan que la gestión de la CE de esta crisis es completamente diferente a cualquier otra y la califican de "única" debido a su envergadura y a sus características específicas: inmediatez, incertidumbre y desinformación.

Por lo tanto, ha obligado a los equipos a ganar agilidad y anticiparse a las preguntas de los usuarios para dar respuestas rápidas y eficaces, y a través de ello, en la mayoría de los casos, se ha reforzado la posición de la organización en el exterior. Durante la crisis se ha dado la situación particular de tener que remitir de manera más asidua y urgente a Salud Pública cualquier información requerida. Según afirma uno de los participantes "ha exigido un rápido aprendizaje y, al mismo tiempo, una respuesta muy constante, adaptada a cada novedad que ofrecía la pandemia". Esto se ha traducido en que en la mayoría de los casos los mensajes se han adaptado a los diversos públicos externos de las organizaciones, transmitiendo la actividad de los centros y la actualidad de la crisis.

Las herramientas de CE más empleadas por los hospitales en esta crisis (gráfico 6) han sido las redes sociales, seguidas de la atención telefónica al paciente, la atención personal y las apariciones en medios. Sin embargo, en un $15,4 \%$ de los casos los centros optaron por el silencio: "No convenía opinar ni la aparición en medios. Había que canalizar la comunicación en Salud Pública".

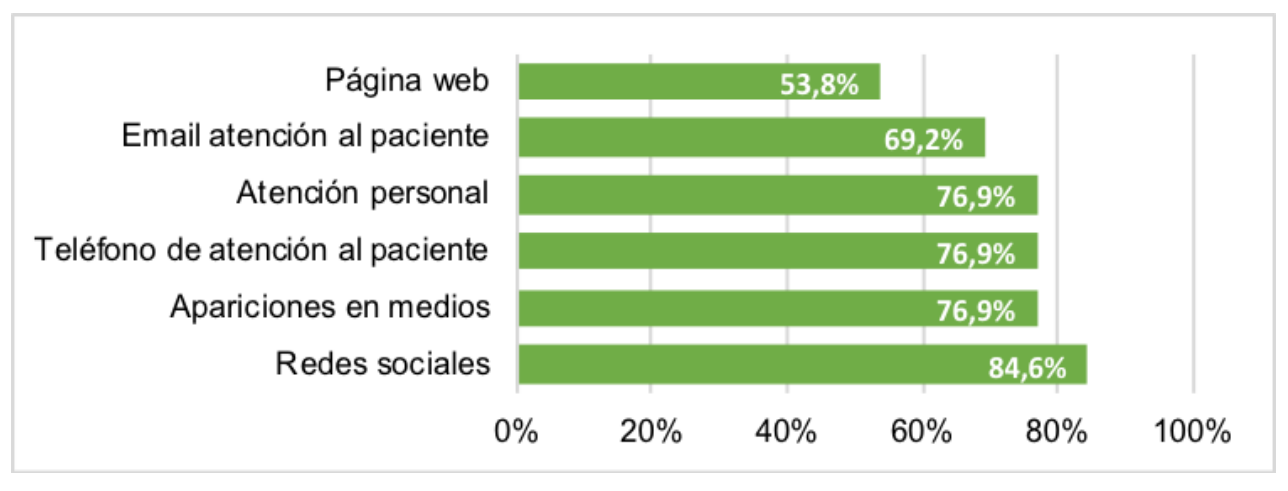

Gráfico 6. Herramientas de CE más empleadas durante la crisis del COVID-19. Fuente: elaboración propia.

El $77 \%$ de las organizaciones ha creado materiales específicos de CE enfocados a la crisis del COVID-19 (tabla 2) como cartelería física y virtual, newsletter y emailing, vídeos informativos o mensajes de la Dirección. 
Gestión de la comunicación interna y externa en instituciones hospitalarias privadas en España durante la crisis del COVID-19

Tabla 2. Materiales de CE creados para gestionar la crisis del COVID-19 en los hospitales.

\begin{tabular}{|l|l|}
\hline Material & Tasa de uso \\
\hline Cartelería física y virtual & $84,6 \%$ \\
\hline Mensajes de la Dirección & $76,9 \%$ \\
\hline Newsletter y emailing & $76,9 \%$ \\
\hline Vídeos informativos & $76,9 \%$ \\
\hline Infografías & $69,2 \%$ \\
\hline Vídeos motivacionales & $53,8 \%$ \\
\hline Folletos informativos & $38,5 \%$ \\
\hline
\end{tabular}

Fuente: elaboración propia.

El feedback que han obtenido de sus stakeholders ha sido positivo en todos los casos. Estos resultados los han obtenido los centros mediante el uso de herramientas propias de medición y en algunos casos han contado con el apoyo de monitores de reputación externa. También "ha sido bien valorado el estar a disposición del Ministerio y/o Consejería de Sanidad para acoger a pacientes Covid y otras cirugías que derivaban los hospitales públicos".

En cuanto a los efectos que esta crisis va a tener sobre la gestión de la CE en el ámbito hospitalario, el $90 \%$ de las respuestas indican que generará cambios por el gran impacto que la pandemia ha tenido en las organizaciones. Se incentivarán la anticipación y proximidad en las comunicaciones y se potenciará el uso de narrativas más simples y cercanas a los intereses de los públicos para lograr que participen en la conversación.

\section{DISCUSIÓN}

La crisis del COVID-19 ha obligado a los sistemas sanitarios a adaptarse para poder enfrentar una situación inédita, tal y como ya han documentado varios estudios (Andrikopoulos \& Johnson, 2020; Shalowitz et al., 2020; Santillán-Doherty, 2020). La comunicación se ha convertido en un factor clave para manejar las crisis sanitarias (Mestre y Bote, 2018; De Sa et al., 2009; WHO, 2005) y en la actual pandemia, en la que se han producido gran cantidad de bulos y noticias falsas (Pérez-Dasilva et al., 2020; Salaverría et al., 2020), se han hecho más necesarias que nunca las fuentes de información fiables, tal y como apuntan Aleixandre-Benavent et al. (2020). Una de estas fuentes de información han sido las instituciones hospitalarias privadas, que han tratado en todo momento de adaptar su Cl y CE (Sánchez, 2011) a la nueva y cambiante realidad, en un periodo de alta incertidumbre y gran desinformación, coincidiendo los resultados obtenidos con otras investigaciones (Carico et al., 2020; Allahverdipour, 2020; Alfonso y Fernández, 2020).

En un contexto en el que el impacto de los mensajes tenía capacidad de afectar no solo a la marca, sino a la red sanitaria en conjunto y a la sociedad en general, los

Revista de Comunicación y Salud, 2020, Vol. 10, no 2, pp. 525-547 
Gestión de la comunicación interna y externa en instituciones hospitalarias privadas en España durante la crisis del COVID-19

hospitales privados han trabajado a partir de sus planes de crisis para aplicar todo tipo de herramientas de comunicación analógica y digital (Medina, 2012; Barquero Cabero, et al., 2018; Costa-Sánchez y López-García, 2020) y crear contenidos específicos con los que informar y atraer a los públicos (Estrella, 2020). Las organizaciones han desarrollado tanto a nivel interno como externo distintas tácticas y han implementado nuevas herramientas que les permitiesen afrontar la crisis sanitaria manteniendo la confianza de sus públicos. Algunas de las que han aplicado y que coinciden con investigaciones anteriores (Edwards et al., 2020; Huanga \& DiStaso, 2020; Abrams \& Greenhawt, 2020; Martín, 2013) son las videoconferencias, la mensajería instantánea, las redes sociales o las apariciones en medios.

\section{CONCLUSIONES}

Este trabajo ha alcanzado sus objetivos al determinar el uso que han hecho de la CE y la $\mathrm{Cl}$ los hospitales privados españoles durante la reciente crisis sanitaria, pudiendo comprobar el empleo de las distintas herramientas y las estrategias que han aplicado hacia sus distintos públicos. Sin embargo, pese a que las entidades estudiadas poseían planes de crisis, estos no contemplaban en la mayoría de los casos un escenario tan extremo como el actual, y tampoco todos los centros disponían de una planificación de las acciones de comunicación a nivel interno. Durante la pandemia se han modificado las rutinas de trabajo de los departamentos de comunicación, que han sacado valiosas lecciones de esta crisis como una mayor agilidad, inmediatez, capacidad de reacción, cohesión entre los miembros de la organización e implicación de los jefes de equipo. Además, se han potenciado herramientas que en circunstancias normales no son de las más empleadas, dado el carácter excepcional de la situación. A nivel interno, y aunque los centros ya disponían de programas de cuidado de empleados, se han incrementado las medidas para proteger la salud de las plantillas y potenciar la seguridad y el sentimiento de pertenencia.

Por otra parte, se aprecia una escasa uniformidad en la situación en el organigrama en los departamentos de $\mathrm{CE}$ y especialmente en los de $\mathrm{Cl}$, que no suelen depender de la Dirección de Comunicación, por lo que pese a su carácter estratégico el área de Comunicación no parece disponer de suficiente autonomía.

En el ámbito hospitalario la comunicación ha sido fundamental para afrontar la crisis del COVID-19 y juega un papel crítico a la hora de encarar las siguientes fases de la pandemia y el periodo de Nueva Normalidad, que llevará aparejados retos hasta ahora desconocidos y que abren nuevos temas de investigación en el área de la comunicación para la salud y la comunicación de crisis.

\section{REFERENCIAS}

Abrams, E. M., \& Greenhawt, M. (2020). Risk communication during COVID-19. The Journal of Allergy and Clinical Immunology: In Practice, 8 (6), 1791-1794. doi: 10.1016/j.jaip.2020.04.012 
Gestión de la comunicación interna y externa en instituciones hospitalarias privadas en España durante la crisis del COVID-19

Acevedo, C. (2020). La gestión de la comunicación de crisis Una tormenta perfecta en nuestra travesía. Iberoamerican Business Journal, 4(1), 100-109. doi: $\underline{10.22451 / 5817 . i b j 2020 . v o l 4.1 .11041}$

Aleixandre-Benavent, R., Castelló-Cogollos, L. y Valderrama-Zurián, J. C. (2020). Información y comunicación durante los primeros meses de Covid-19. Infodemia, desinformación y papel de los profesionales de la información. Profesional de la información, v. 29, n. 4, e290408. doi: 10.3145/epi.2020.jul.08

Almada, A. A. (2009). Marketing de crisis, Herramientas concretas para afrontar la actual crisis económica. Madrid: Pirámide.

Alfonso Sánchez, I., \& Fernández Valdés, M. (2020). Comportamiento informacional, infodemia y desinformación durante la pandemia de COVID-19. Anales De La Academia De Ciencias De Cuba, 10(2), e882. Recuperado de http://www.revistaccuba.sld.cu/index.php/revacc/article/view/882/889

Allahverdipour H. (2020) Global Challenge of Health Communication: Infodemia in the Coronavirus Disease (COVID-19) Pandemic. J Educ Community Health, 7 (2) :65-67. URL: http://jech.umsha.ac.ir/article-1-1000-en.html

Andrikopoulos, S. \& Johnson, G. (2020) The Australian response to the COVID-19 pandemic and diabetes - Lessons learned. Diabetes Research and Clinical Practice, doi: $\underline{10.1016 / j . d i a b r e s .2020 .108246}$

Barquero, J. D. y Barquero, M. (2005). Manual de Relaciones Públicas, Comunicación y Publicidad. Barcelona: Editorial Gestión 2000.

Barquero Cabero, M., Rodríguez Terceño, J. y Gonzálvez Vallés, J. E. (2018). Tecnologías de la Comunicación y posverdad: implicaciones para la gestión de la Comunicación Hospitalaria. Revista de Comunicación y Salud, 8(1), 85-97. doi: 10.35669/revistadecomunicacionysalud.2018.8(1).85-97

Bustamante, E. (2012). La promoción de la salud desde la comunicación interna. $\begin{array}{llll}\text { Revista de Comunicación y } & \text { Salud, }\end{array}$ doi: $10.35669 /$ revistadecomunicacionysalud.2012.2(2).79-90

Calvo-Calvo, M.A. (2016). La comunicación de crisis en errores sanitarios: reflexiones sobre las estrategias institucionales. Index de Enfermería, 25(3), 161-165. Recuperado de http://scielo.isciii.es/scielo.php?script=sci arttext\&pid=S1132$12962016000200008 \& \operatorname{lng}=$ es\&tlng=es.

Calleja-Reina, M. A., Paniagua Rojano, F. J, y Victoria Mas, J. S. (2018). Herramientas digitales y Comunicación de Crisis: El papel de las redes sociales según la voz de los expertos (2015). Estudios sobre el Mensaje Periodístico, 24 (2), 1147-1167. doi: 10.5209/ESMP.62206 
Gestión de la comunicación interna y externa en instituciones hospitalarias privadas en España durante la crisis del COVID-19

Carico, R.R., Sheppard, J. \& Thomas, C.B. (2020) Community pharmacists and communication in the time of COVID-19: Applying the health belief model. Research in Social and Administrative Pharmacy. doi: 10.1016/j.sapharm.2020.03.017

Castillo Esparcia, A. (2010). Introducción a las Relaciones Públicas. Málaga: Editorial Instituto de Investigación en Relaciones Públicas.

Costa, C. (2011). La comunicación en el hospital: la gestión de la comunicación en el ámbito sanitario. Sevilla: Comunicación social, ediciones y publicaciones.

Costa-Sánchez, C. y López-García, X. (2020). Comunicación y crisis del coronavirus en España. Primeras lecciones. El profesional de la información, 29(3), e290304. doi: 10.3145/epi.2020.may.04

Costa-Sánchez, C., Túñez-López, M. y Videla-Rodríguez, J.J. (2016). "Hospitales españoles en la web social. Gestión de Facebook y Twitter por el Hospital Sant Joan de Dèu (Barcelona)". Revista Latina de Comunicación Social, 71, 1.108-1.130. doi: $\underline{10.4185 / R L C S-2016-1137}$

Danet, A., García Romera, I. y March Cerdà, J. C. (2016). Liderazgo transformacional en las organizaciones sanitarias. Una revisión bibliográfica. Revista de Comunicación y Salud, 6, 83-97. doi: 10.35669/revistadecomunicacionysalud.2016.6(1).83-97

De Sa, J., Mounier-Jack, S., \& Coker, R. (2009). Risk communication and management in public health crises. Public health, 123(10), 643-644. doi: $10.1016 /$ j.puhe.2009.07.017

Edwards J. A., Breitman I., Kovatch I., Dresner L., Smith T.Y., Brunicardi F.C. \& Schwartzman A. (2020). Lessons Learned at a COVID-19 designated hospital, The American Journal of Surgery. doi: 10.1016/j.amjsurg.2020.07.029

Estrella, E. C. M. (2020). Uso de personajes y metáforas en la gestión de la crisis sanitaria del COVID-19. Revisión de la comunicación de sanidad pública en España y México. Revista española de Comunicación en Salud, 1, 319-327. doi: $\underline{10.20318 / \text { recs.2020.5458 }}$

García-Hernández, M.L, Martínez-Rodrigo, E. y Victoria Mas, J. S. (2016) Comunicación corporativa-RSC-Divulgación científica. Propuesta de "triángulo virtuoso" para las empresas biotecnológicas. Observatorio (OBS*) Journal, 10 (4), 056-076. Recuperado de http://www.scielo.mec.pt/scielo.php?script=sci arttext\&pid=S1646$\underline{59542016000500004 \& \operatorname{lng}=\mathrm{pt} \& \ln \mathrm{g}=\mathrm{es}}$

González Pacanowski, A.; Medina Aguerrebere, P. y Iglesias García, M. (2017). Estrategia para la optimización de la comunicación de riesgo en la crisis del Ébola. Revista de Comunicación y Salud, vol. 7, 173-186. Recuperado de http://revistadecomunicacionysalud.org/index.php/rcys/article/view/123 
Gestión de la comunicación interna y externa en instituciones hospitalarias privadas en España durante la crisis del COVID-19

Gost Garde, J. (2015). Difusión de los resultados en salud de los hospitales. Anales del Sistema Sanitario de Navarra, 38(2), 181-184. doi: 10.4321/S1137$\underline{66272015000200001}$

Grover, S., Dua, D., Sahoo, S., Mehra, S.A., Nehra, R. \& Chakrabarti, S. (2020). Why all COVID-19 hospitals should have mental health professionals: The importance of mental health in a worldwide crisis! Asian Journal of Psychiatry, 51, 102147. doi: $\underline{10.1016 / j . a j p .2020 .102147}$

Hernández, R., Fernández, C. y Baptista P. (2010). Metodología de la Investigación. Distrito Federal de México, México: McGraw Hill.

Huanga,Y \& DiStaso, M. (2020). Responding to a Health Crisis on Facebook: The Effects of Response Timing and Message Appeal, Public Relations Review, 46, doi: $\underline{10.1016 / \text { i.pubrev.2020.101909 }}$

Igartua, J. J. (2011). Mejor convencer entreteniendo: comunicación para la salud y persuasión narrativa. Revista de Comunicación y Salud, 1(1), 73-87. doi: $\underline{10.35669 / \text { revistadecomunicacionysalud.2011.1(1).73-87 }}$

Igartua, J.J., Ortega-Mohedano, F. y Arcila-Calderón, C. (2020). Communication use in the times of the coronavirus. A cross-cultural study. El profesional de la información, 29(3), e290318. doi: 10.3145/epi.2020.may.18

Kortepeter, M. G., Kwon E.H., Christopher, G. W., Hewlett A.L. \& Cieslak, T. J. (2017). Interagency cooperation is the key to an effective pandemic response, The Lancet Infectious Diseases, 17(1), 21. doi: 10.1016/S1473-3099(16)30549-7

Lázaro-Rodríguez, P. y Herrera-Viedma, E. (2020). Noticias sobre Covid-19 y 2019nCoV en medios de comunicación de España: el papel de los medios digitales en tiempos de confinamiento. El profesional de la información, 29(3), e290302. doi: 10.3145/epi.2020.may.02

Losada, J. C. (2018). La comunicación en la gestión de crisis sanitarias: aplicación del modelo de (NO) CRISIS al contagio del virus del ébola en España. ESTRATEGAS, Investigación en Comunicación ISSN: 2550-6870, Vol. 5. Recuperado de http://marketing.udla.edu.ec/ojs/index.php/estrategas/article/view/150/134

Lu, X. \& Jin, Y. (2020). Information vetting as a key component in social-mediated crisis $\mathrm{T}$ communication: An exploratory study to examine the initial conceptualization. Public Relations Review, 46(2). doi: 10.1016/i.pubrev.2020.101891

Luecke, L. (2005). Gestión de crisis convertirlas en oportunidades. Barcelona: Ediciones Deusto. 
Gestión de la comunicación interna y externa en instituciones hospitalarias privadas en España durante la crisis del COVID-19

March Cerdá, J. C. (2011). El riesgo de una mala comunicación de riesgos. Revista de Comunicación y Salud, 61-66. doi: $\underline{10.35669 / \text { revistadecomunicacionysalud.2011.1(2).61-66 }}$

Maniya O. Z., \& McGreevy, J. (2020). Managing Emergency Department Risk Through Communication and Documentation. Emergency Medicine Clinics of North America, 38(2), 267-281. doi: 10.1016/..emc.2020.01.007

Martín, P. G. (2013). ¿Crisis sanitarias o crisis mediáticas? Quaderns de la Fundació Dr. Antoni Esteve, 25, 39-46. Recuperado de https://www.raco.cat/index.php/QuadernsFDAE/article/download/278649/366395

Masip, P., Aran-Ramspott, S., Ruiz-Caballero, C., Suau, J., Almenar, E. y PuertasGraell, D. (2020). Consumo informativo y cobertura mediática durante el confinamiento por el Covid-19: sobreinformación, sesgo ideológico y sensacionalismo. El profesional de la información, 29(3), e290312. doi: 10.3145/epi.2020.may.12

Medina Aguerrebere, P. (2012). El valor estratégico de la comunicación interna hospitalaria. Revista de Comunicación y Salud, 2(1), 19-28. doi: $10.35669 /$ revistadecomunicacionysalud.2012.2(1).19-28

Mestre Ortega, P. y Bote Ruiz de Gordoa, J. J. (2018). La gestión de la comunicación institucional en los casos de fiebre hemorrágica de Crimea-Congo en la Comunidad de Madrid. Propuesta de decálogo de comunicación de crisis de salud pública. Revista de Comunicación y Salud, 8(1), 71-84. doi: 10.35669/revistadecomunicacionysalud.2018.8(1).71-84

Micaletto Belda, J. (2018). Principios fundamentales de la comunicación en crisis: una configuración táctica. Razón Y Palabra, 22(2_101), 503-517. Recuperado de http://www.revistarazonypalabra.org/index.php/ryp/article/view/1079

Micaletto Belda, J. P. y Gallardo Vera, L. (2015). La comunicación institucional en la crisis del ébola en Europa: el caso de la crisis española de 2014 en sus inicios. Revista Internacional de Relaciones Públicas. 5(9), 89-110. doi: 10.5783/RIRP-92015-06-89-110

Mira, J.J. (2020). Pandemia COVID-19: y ahora ¿qué? Journal of Healthcare Quality Research, 35(3):133-135. doi: 10.1016/j.jhgr.2020.04.001

Mira, J.J., Lorenzo, S., Navarro, I.M., Guilabert, M., y Pérez-Jover, V. (2015). La reputación de los hospitales españoles: bases para el desarrollo de un índice de reputación de los hospitales. Anales del Sistema Sanitario de Navarra, 38(2), 247254. doi: $10.4321 / \mathrm{S} 1137-66272015000200008$ 
Gestión de la comunicación interna y externa en instituciones hospitalarias privadas en España durante la crisis del COVID-19

Pérez-Dasilva, J.A., Meso-Ayerdi, K. y Mendiguren-Galdospín, T. (2020). Fake news y coronavirus: detección de los principales actores y tendencias a través del análisis de las conversaciones en Twitter. El profesional de la información, 29(3), e290308. doi: 10.3145/epi.2020.may.08

Prieto, M. D. M., y Olmo, F. J. V. (2010). El plan de comunicación hospitalario: herramienta de gestión sanitaria. Editorial club universitario.

Rodríguez Andrés, R. (2011). La efectividad del uso del miedo como factor persuasivo en la comunicación de riesgos en las crisis sanitarias. Revista de Comunicación y Salud, 1 (2), 33-46. doi: 10.35669/revistadecomunicacionysalud.2011.1(2).33-46

Rodríguez González, M. del M., Marauri Castillo, I., Armentia Vizuet, J. I. y Marín Murillo, F. (2020). De la crisis del pepino a la crisis del huevo. Aciertos y errores en la gestión de comunicación de crisis alimentarias. Revista de Comunicación y Salud, 10(1), 91-114. doi: $10.35669 /$ rcys.2020.10(1).91-114

Sánchez, C. C. (2011). La comunicación en el hospital: la gestión de la comunicación en el ámbito sanitario (Vol. 42). Zamora: Comunicación Social. https://hdl.handle.net/10171/56925

Sanford, S., Schwartz, B. \& Khan, Y. (2020). The role of tacit knowledge in communication and decision-making during emerging public health incidents. International Journal of Disaster Risk Reduction, 50, 101681. doi: $\underline{10.1016 / j . i j d r r .2020 .101681}$

Santillán-Doherty, (2020). Pandemia de COVID-19: preparando la lucha contra la peste del siglo XXI. Neumología y Cirugía de Tórax, 79 (1), 4-7. doi: $\underline{10.35366 / 93422}$

Salaverría, R., Buslón, N., López-Pan, F., León, B., López-Goñi, I. y Ervti, M.C. (2020). Desinformación en tiempos de pandemia: tipología de los bulos sobre la Covid-19. El profesional de la información, 29(3), e290315. doi: 10.3145/epi.2020.may.15

Shalowitz, D.I., Lefkowits, C., Landrum, L.M., Von Gruenigen, V.E. \& Spillman, M. A. (2020). Principles of ethics and critical communication during the COVID- 19 pandemic, Gynecologic Oncology, 158 (3), 526-530. doi: 10.1016/j.ygyno.2020.06.494

Stajnolovic, M. (2015). Percepción social de riesgo: una mirada general y aplicación a la comunicación de salud. Revista de Comunicación y Salud, 5, 99-110. doi: 10.35669/revistadecomunicacionysalud.2015.5(1).99-110

Teddlie, C. \& Abbas, T. (2010). Overview of Contemporary Issues in Mixed Methods Research. En T. Abbas, \& C. Teddlie (Eds.), Handbook of Mixed Methods in Social and Behavioral Research (pp. 1-41). doi: 10.4135/9781506335193.n1 
Gestión de la comunicación interna y externa en instituciones hospitalarias privadas en España durante la crisis del COVID-19

Voulgaris A., Ferini-Strambi L. \& Steiropoulos, P. (2020). Sleep medicine and COVID-

19. Has a new era begun?. Sleep Medicine, 73, 170-176. doi: 10.1016/j.sleep.2020.07.010

Wimmer, R. D. \& Dominick, J. R. (1996). La investigación científica de los medios de comunicación: una introducción a sus métodos. Bosch.

World Health Organization (WHO). (2005). WHO outbreak communication guidelines. Geneva: WHO. Recuperado de https://www.who.int/csr/resources/publications/WHO CDS 2005 28/en/

Xifra, J. (2020). Comunicación corporativa, relaciones públicas y gestión del riesgo reputacional en tiempos del Covid-19. El profesional de la información, 29(2), e290220. doi: $\underline{10.3145 / e p i .2020 . m a r .20}$

Xifra, J. (2009). Comunicación proactiva. La gestión de conflictos potenciales en las organizaciones. Barcelona: Editorial Gedisa, S.A.

Zhao, X., Zhan, M., \& Ma, L. (2020). How publics react to situational and renewing organizational responses across crises: Examining SCCT and DOR in socialmediated crises. Public Relations Review, 46(4), 101944. doi: 10.1016/i.pubrev.2020.101944

\section{AUTORES}

\section{Andrea Castro-Martínez}

Docente e investigadora en la Universidad de Málaga desde 2016. Anteriormente fue durante ocho años subdirectora de los grados de Comunicación (Periodismo y Publicidad y RRPP), Diseño Gráfico y Diseño de Interiores en EADE, sede de la Universidad de Gales en Málaga, donde también era docente de los grados y del MBA. Licenciada en Comunicación Audiovisual y en Publicidad y Relaciones Públicas, ha cursado varios expertos en comunicación y marketing y un MBA. Ha trabajado en diferentes medios de comunicación y ha sido docente y consultora de Marketing, Publicidad y Comunicación. Sus líneas de investigación se centran en relaciones públicas, comunicación estratégica y comunicación interna y en nuevas formas publicitarias.

Orcid ID: https://orcid.org/0000-0002-2775-625X

Google Scholar: https://scholar.google.com/citations?user=Zj4rXMMAAAAJ\&hl=es

Redalyc: $h$ ttps://www.redalyc.org/autor.oa?id=39547

Researchid: $\mathrm{AAH}-4909-2020$

Researchgate: https://www.researchgate.net/profile/Andrea Castro-Martinez

Academia.Edu: https://uma.academia.edu/AndreaCastroMartinez

Scopus Id: https://www.scopus.com/authid/detail.uri?authorld=57210469485

Dialnet: https://dialnet.unirioja.es/servlet/autor?codigo=4815030 
Gestión de la comunicación interna y externa en instituciones hospitalarias privadas en España durante la crisis del COVID-19

\section{Pablo Díaz-Morilla}

Investigador en la Universidad de Málaga. Es director y docente en los Grados en Comunicación (Periodismo y Publicidad y RRPP) en EADE University of Wales in Málaga desde 2007 y en las Enseñanzas artísticas superiores en Diseño Gráfico, de Interiores y de Producto desde 2014. Imparte clases también en el MBA. Doctorando en Comunicación por la UMA, también es dramaturgo y guionista, con trabajos y textos premiados a nivel nacional. Su línea de investigación se centra en comunicación comercial, nuevas formas publicitarias, teatro y productos culturales y en relaciones públicas.

Orcid Id: https://orcid.org/0000-0002-7914-3391

Google Scholar: https://scholar.google.es/citations?user=Rleeh7gAAAAJ\&hl=es\&oi=ao Researchid: AAW-4812-2020

Researchgate: https://www.researchgate.net/profile/Pablo Diaz Morilla

Academia.Edu: independent.academia.edu/PabloDíazMorilla

Redalyc: https://www.redalyc.org/autor.oa?id=39548 